\title{
1 Full-title
}

2 Lack of parent-of-origin effects in Nasonia jewel wasp: a replication and extension study

\section{Short-title}

$5 \quad$ Nasonia parent-of-origin effects

6

\section{$7 \quad$ Authors}

8 Kimberly C. Olney ${ }^{1,2}$, Joshua D. Gibson ${ }^{3}$, Heini M. Natri ${ }^{2}$, Avery Underwood $^{1,2}$, Juergen Gadau ${ }^{4}$,

9 Melissa A. Wilson ${ }^{1,2,5^{*}}$

10

\section{Affiliations}

12 1. School of Life Sciences, Arizona State University, Tempe AZ 85281 USA

13 2. Center for Evolution and Medicine, Arizona State University, Tempe AZ 85281 USA

14 3. Department of Biology, Georgia Southern University, Statesboro GA 30460 USA

15 4. Institut fuer Evolution and Biodiversity, University of Muenster, Muenster, Germany 48149

16 5. Center for Mechanisms of Evolution, The Biodesign Institute, Arizona State University, Tempe

$17 \quad$ AZ 85281 USA

${ }^{*}$ Corresponding author

20 Melissa A. Wilson

21 School of Life Sciences | Arizona State University | PO Box 874501| Tempe, AZ 85287-4501

22 mwilsons@asu.edu

\section{Author contributions}

25 KCO: Supervision, Formal Analysis, Investigation, Visualization, Writing - Original Draft

26 Preparation, Writing - Review and Editing 
27 JDG: Conceptualization, Supervision, Formal Analysis, Investigation, Resources, Experimental

28 Procedures, Writing - Original Draft Preparation, Writing - Review and Editing, Visualization

29 HMN: Supervision, Formal Analysis, Investigation, Visualization, Writing - Original Draft

30 Preparation, Writing - Review and Editing

31 AU: Formal Analysis, Investigation, Writing - Review and Editing

32 JG: Conceptualization, Funding acquisition, Supervision, Resources, Writing - Review and Editing

33 MAW: Conceptualization, Supervision, Visualization, Resources, Project Administration, Writing

34 - Original Draft Preparation, Writing - Review and Editing, Funding Acquisition

\section{Abbreviations}

37 Allele-specific expression (ASE)

38 Differential expression (DE)

39 Differentially expressed genes (DEGs)

40 False discovery rate (FDR)

$41 \log _{2}$ fold-change ( $\left.\log _{2} F C\right)$

$42 \log$ counts per million (logCPM)

43 Nasonia giraulti maternal and paternal (GG)

$44 \quad$ Nasonia vitripennis maternal and paternal (VV)

$45 \quad$ N. giraulti maternal, N. vitripennis paternal (GV) $F_{1} G V$

$46 \quad$ N. vitripennis maternal, N. giraulti paternal (VG) $F_{1} V G$

$47 \quad$ No difference (ND)

48 RNA sequence (RNAseq)

49 Standard deviation (SD)

50 Variant call format (VCF) 


\section{Abstract}

52 In diploid cells, the paternal and maternal alleles are, on average, equally expressed. There are

53 exceptions from this: a small number of genes express the maternal or paternal allele copy

54 exclusively. This phenomenon, known as genomic imprinting, is common among eutherian

55 mammals and some plant species; however, genomic imprinting in species with haplodiploid sex

56 determination is not well characterized. Previous work reported no parent-of-origin effects in the

57 hybrids of closely related haplodiploid Nasonia vitripennis and Nasonia giraulti jewel wasps,

58 suggesting a lack of epigenetic reprogramming during embryogenesis in these species. Here, we

59 replicate the gene expression dataset and observations using different individuals and

60 sequencing technology, as well as reproduce these findings using the previously published RNA

61 sequence data following our data analysis strategy. The major difference from the previous

62 dataset is that they used an introgression strain as one of the parents and we found several loci

63 that resisted introgression in that strain. Our results from both datasets demonstrate a species-

64 of-origin effect, rather than a parent-of-origin effect. We present a reproducible workflow that

65 others may use for replicating the results. Overall, we reproduced the original report of no parent-

66 of-origin effects in the haplodiploid Nasonia using the original data with our new processing and

67 analysis pipeline and replicated these results with our newly generated data.

\section{Introduction}

69 Parent-of-origin effects occur when there is a biased expression (or completely monoallelic

70 expression) of alleles inherited from the two parents [1,2]. Monoallelic gene expression in the

71 offspring is hypothesized to be primarily the result of genetic conflict between parents over

72 resource allocation in the offspring $[3,4]$. In mammals, the mechanism of these parent-of-origin

73 effects occurs via inherited methylation of one allele $[1,5]$. In insects, the relationship between

74 methylation of genomic DNA and the expression of the gene that it encodes is not as well 
75 characterized but studies of social insects showed that there is a positive correlation of DNA

76 methylation of gene bodies and gene expression [6].

Honey bees have been a focal group for investigation of parent-of-origin effects in insects due to differences in the kinship between queens, males, and workers $[7,8]$. Multiple mating by queens results in low paternal relatedness between workers and should lead to intragenomic conflict over worker reproduction (laying unfertilized eggs to produce males), and ultimately should favor the

82 biased expression of paternal alleles that promote worker reproduction [9]. Utilizing a cross

83 between European (Apis mellifera ligustica) and Africanized honey bees, Galbraith et al. 2016

84 identified genes exhibiting a pattern of biased paternal allele overexpression in worker

85 reproductive tissue from colonies that were queenless and broodless, a colony condition that promotes worker reproduction [9]. Smith et al. 2020 found a similar pattern of paternal allele overexpression in diploid (worker-destined) eggs in a cross between two African subspecies, A.m. scutellate and A.m. capensis [10]. In reciprocal crosses of European (A.m. ligustica and A.m. carnica) and Africanized honey bees reared in colonies containing both brood and a queen, Kocher et al. 2015 instead found parent-of-origin effects in gene expression that were largely 91 overexpressing the maternal allele in both directions of the cross [11]. These studies provide 92 evidence for parent-of-origin effects in the honey bee, a eusocial Hymenoptera. The Kocher et al. 932016 dataset also exhibited asymmetric maternal allelic bias in which the paternal allele was 94 silenced, but only in hybrids with Africanized fathers [12]. This set of biased genes was enriched 95 for mitochondrial-localizing proteins and is overrepresented in loci associated with aggressive 96 behavior in previous studies [13,14]. Interestingly, these same crosses exhibit high aggression in 97 the direction of the cross with the Africanized father but not in the reciprocal cross [15], and 98 aggression and brain oxidative metabolic rate appears to be linked in honey bees [16]. This study 99 points toward a potential role of allelic bias and nuclear-mitochondrial genetic interactions in wide 100 crosses of honey bees. 
102 The parasitoid wasp genus Nasonia has emerged as an excellent model for studying genomic 103 imprinting in Hymenoptera. Like honey bees and all Hymenoptera, Nasonia has a haplodiploid 104 sex-determination system in which females are diploid, developing from fertilized eggs, and males 105 are haploid, developing from unfertilized eggs. However, it serves as a strong contrast to studying 106 parent-of-origin effects in the eusocial Hymenoptera as Nasonia is solitary and singly-mated, 107 which should result in less genomic conflict and therefore less selective pressure for genomic 108 imprinting based on kinship. By studying allelic expression biases in this system, we can better 109 assess genomic imprinting in the absence of kin selection and the potential contribution of 110 nuclear-mitochondrial interactions to biased allelic expression. Nasonia is well-suited for these 111 kinds of studies as two closely related species of Nasonia - N. vitripennis and $N$. giraulti - that 112 diverged $\sim 1$ million years ago (Mya) and show a synonymous coding divergence of $\sim 3 \%$ [17], can 113 still produce viable and fertile offspring [18]. Highly inbred laboratory populations of $N$. vitripennis 114 and $N$. giraulti with reduced polymorphism provide an ideal system for identifying parent-of-origin 115 effects in hybrid offspring [19]. However, the species do show genetic variation and 116 incompatibilities, such that recombinant F2 males (from unfertilized eggs of F1 hybrid females) 117 suffer asymmetric hybrid breakdown in which $50 \%$ to $80 \%$ of the offspring die during development 118 [18]. The mortality is dependent on the direction of the cross and those with N. giraulti maternity 119 (cytoplasm) have the highest level of mortality. Nuclear-mitochondrial incompatibilities have been 120 implicated in this and candidate loci have been identified [20-22]. Despite this high level of 121 mortality in F2 males, there is no obvious difference in mortality of the F1 mothers of these males 122 and non-hybrid females, further highlighting this as an excellent system in which to test the 123 potential role of allelic expression bias in mitigating hybrid dysfunction.

125 Wang et al. 2016 used genome-wide DNA methylation and transcriptome-wide gene expression 126 data from 11 individuals to test whether differences in DNA methylation drive the differences in 
gene expression between $N$. vitripennis and $N$. giraulti, and whether there are any parent-of-origin effects (parental imprinting and allele-specific expression) [19]. They used reciprocal crosses of these two species and found no parent-of-origin effects, suggesting a lack of genomic imprinting. Unlike the work in honey bees, however, there have not been multiple independent investigations of evidence for parent-of-origin effects in Nasonia.

Reproducibility is a major concern in science, particularly for the biological and medical sciences $[23,24]$. To replicate is to make an exact copy. To reproduce is to make something similar to

135 something else. Reports have shown that significant factors contributing to irreproducible 136 research include selective reporting, unavailable code and methods, low statistical power, poor 137 experimental design, and raw data not available from the original lab $[23,25,26]$. In RNAseq experiments, raw counts are transformed into gene or isoform counts, which requires an in silico bioinformatics pipeline [27]. These pipelines are modular and parameterized according to the experimental setup [27]. The choice of software, parameters used, and biological references can

141 alter the results. In RNAseq, filters can also improve the robustness of differential expression calls and consistency across sites and platforms [28]. There is no, and there may never be, a defined optimal RNAseq processing pipeline from raw sequencing files to meaningful gene or isoform

144 counts. Thus, the same data can be processed in a multitude of ways by the choice of software, 145 parameters, and references used [27]. Given the exact same inputs, software, and parameters, 146 one can reproduce the analysis if the authors provide this documentation and make explicit the 147 information related to the data transformation used to the RNAseq data [27]. In the case of the 148 Wang et al. 2016, the methods and experimental design were exceptionally well documented, 149 and the authors made available their raw data [19].

151 To address whether the Wang et al. 2016 findings of lack of parent-of-origin effects in Nasonia 152 may be replicated and reproduced, we conducted two sets of analyses. We first downloaded the 
153 raw data from 11 individuals [19] and replicated differential expression (DE) and allele-specific

154 expression (ASE) analyses. This allowed us to characterize species differences in gene 155 expression, hybrid effects relative to each maternal and paternal line, and possible parent-of156 origin effects using new alignment methods and software. Second, we reproduced the 157 experimental setup with new individuals, generated transcriptome-wide expression levels of 12 158 Nasonia individuals (parental strains and reciprocal hybrids), named here as the Wilson data 159 using similar, but not identical strains as the Wang et al. 2016 samples, which we named as the 160 R16A Clark data. The Wilson data, reported here, used the standard N. giraulti strain (RV2Xu). 161 The R16A Clark N. giraulti differs from the RV2Xu strain in that it has a nuclear N. giraulti genome 162 introgressed into a N. vitripennis cytoplasm which harbour N. vitripennis mitochondria. Both 163 studies used the same highly inbred standard N. vitripennis strain, ASymCx. We completed the 164 above analyses to test for robust reproducibility in biased allele and parent-of-origin effects in 165 Nasonia. In this analysis, we processed both the R16A Clark and Wilson data using the same 166 software and thresholds, starting with the raw FASTQ files. While we detect some differences in 167 the specific differentially expressed genes between the two datasets, our study reproduces and 168 confirms the main conclusions of the Wang et al. 2016 study: we observe similar trends in the DE 169 and ASE genes, and we detect no parent-of-origin effects in Nasonia hybrids, indicating a 170 validation of the lack of epigenetic reprogramming during embryogenesis in this taxa [19]. We 171 make available the bioinformatics processing and analysis pipeline used for both the R16A Clark 172 and Wilson datasets for easily replicating the results reported here: 173 https://github.com/SexChrLab/Nasonia. Finally, during the process of reproducing these results, 174 we extend them to show potential interactions between the mtDNA and autosomal genome that 175 were not apparent in the original study. 


\section{Results}

\section{Samples cluster by species and hybrid in R16A Clark and Wilson datasets}

178 We used Principal Component Analysis (PCA) of gene expression data to explore the overall

179 structure of the two datasets, R16A Clark and Wilson. Although the reciprocal hybrids from the

180 two datasets are slightly different Fig 1B, in both sets, samples from the two species (strains)

181 form separate clusters, with the clustering of the hybrid samples between them Fig $2 \mathrm{~A}$. The first

182 PC explains most of the gene expression variation in both datasets, with proportions of variance

183 explained $58.17 \%$ in R16A Clark and $61.69 \%$ in the Wilson data. Further, despite differences in

184 experimental protocols, the transcriptome-wide gene expression measurements across the

185 different crosses and species are highly correlated between the R16A Clark and Wilson dataset,

186 Fig 3. There is a difference in the mean RNAseq library size between the two datasets. The mean

187 RNAseq library size for the R16A Clark samples is $48,893,872$ base pairs (bp) $(S D=11,603,536)$

188 and the Wilson samples is $16,518,955 \mathrm{bp}(\mathrm{SD}=3,205,303)$, S1 Table. Overall, we observe that

189 most of the variation in the data is explained by species and hybrids. 


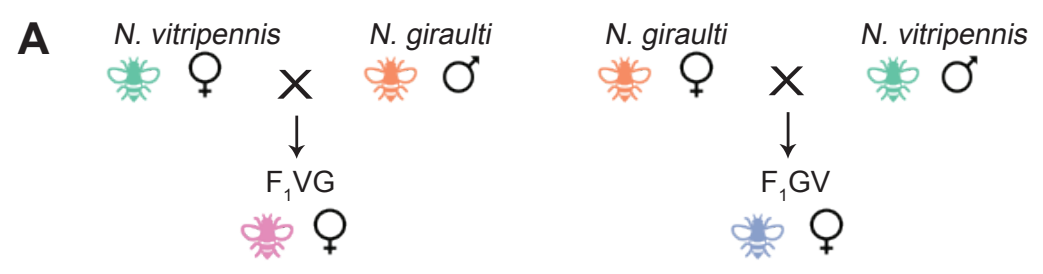

B

Clark

Wilson

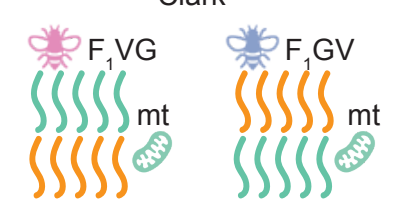

C

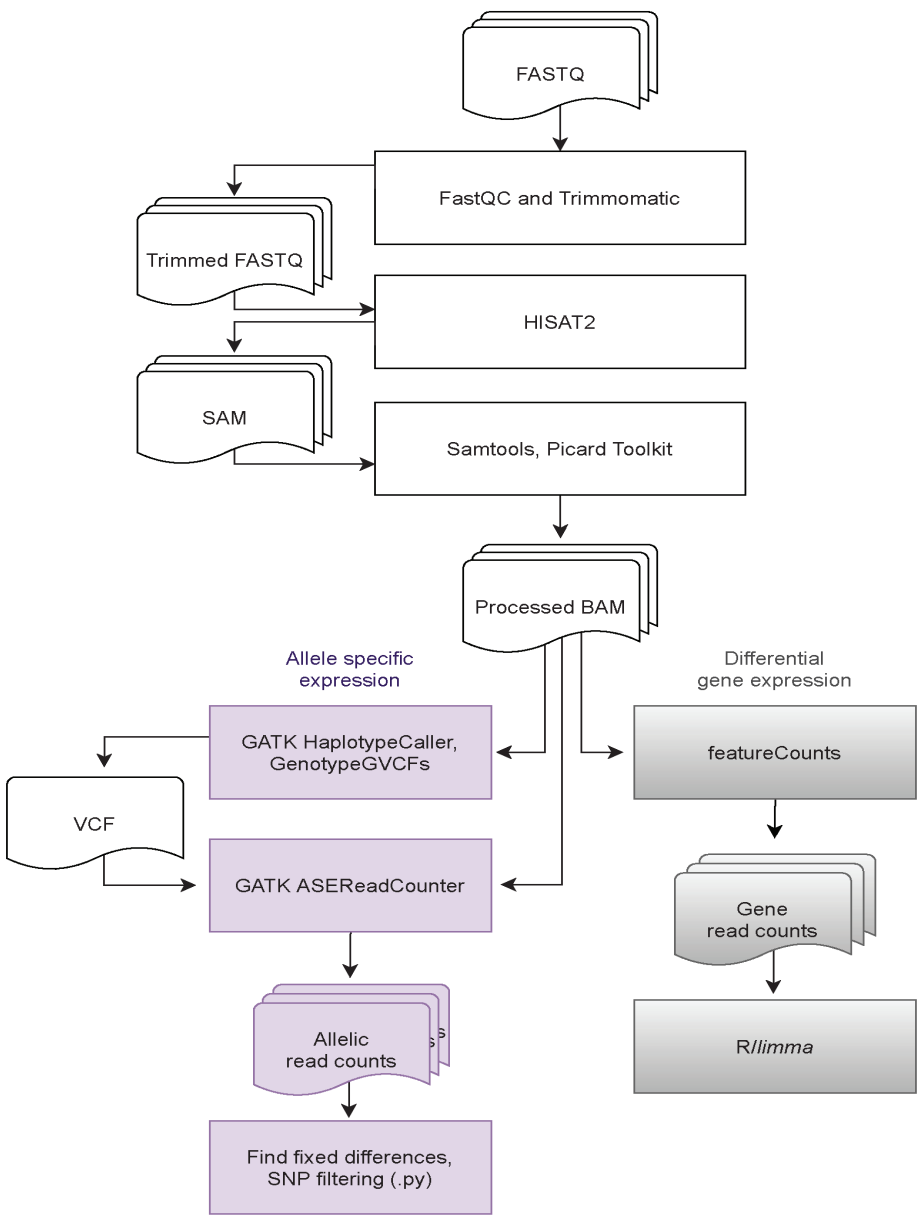

191 Fig 1. A: A schematic illustration of the reciprocal $F_{1}$ crosses. B: Schematic illustration of the 192 hybrids nuclear and mitochondrial genomic make up. All hybrids are heterozygous at every 193 nuclear locus for their two parent's alleles. The R16A Clark hybrids have $N$. vitripennis 194 mitochondria, regardless of maternal species. The Wilson hybrids have their maternal species 195 mitochondria. C: Overview of the data processing and analysis workflow. 

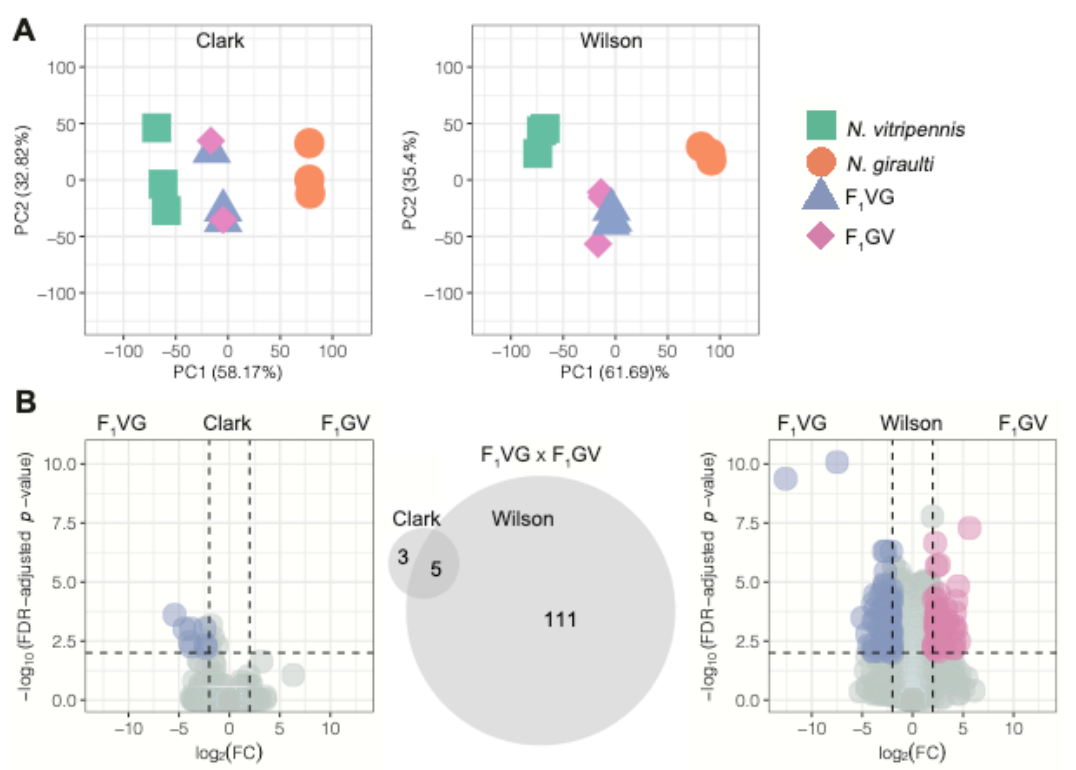

198 Fig 2. A: Gene expression PCA based on all expressed genes (mean FPKM $\geq 0.5$ across three

199 biological replicates in at least one sample group) in the R16A Clark and Wilson datasets when

200 taking the average between the $N$. vitripennis and pseudo $N$. giraulti reference genomes. B:

201 Volcano plots of differentially expressed genes between the two reciprocal hybrids in the R16A

202 Clark and Wilson datasets. Significance thresholds of an FDR-adjusted $p$-value $\leq 0.01$ and an

203 absolute $\log _{2} F C \geq 2$ are indicated. A Venn diagram shows the overlap of the significant DEGs. 

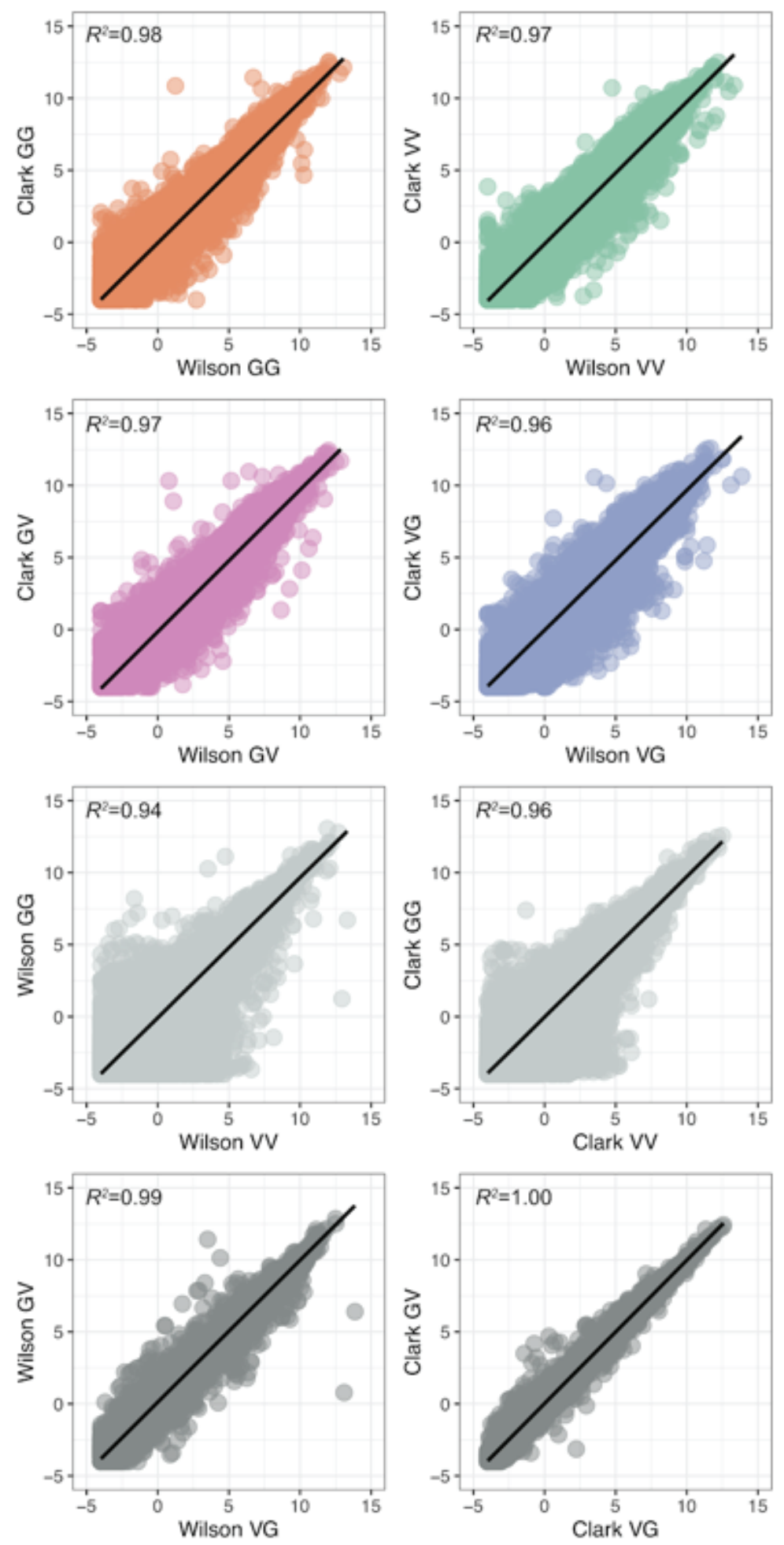

Fig 3. Gene expression correlation between the Wilson and R16A Clark datasets, as well as between species and between reciprocal hybrids within each dataset. Mean logCPM expression of each quantified gene in each cross and dataset is shown. Pearson's correlation $R^{2}$ is indicated. 


\section{vitripennis and $\mathbf{N}$. giraulti}

210 We detect more differentially expressed genes (DEGs) in the Wilson dataset despite the smaller

211 library sizes, particularly in the comparison involving the hybrid samples (Fig 2B). We called

212 DEGs, FDR $\leq 0.01$, and absolute $\log _{2}$ fold change $\geq 2$, between the different species and crosses

213 within both datasets (Fig 2B and S1 Fig). In the N. vitripennis (VV) x N. giraulti (GG) comparison,

214 we identify 799 and 1,001 DEGs in the R16A Clark and Wilson datasets, respectively. We observe

215 a $45.5 \%$ overlap of these DEGs between the datasets (S1 Fig). As expected, we detect fewer

216 DEGs in the comparisons involving the hybrids (Fig 1B). We detect only small differences in the

217 numbers of DEGs called in the R16A Clark and Wilson datasets when examining hybrid effects

218 relative to each maternal line (S1 Fig). However, these DEGs show little overlap between the

219 datasets, with the proportions of overlapping DEGs in VVxVG, VVxGV, GGxVG, and GGxGV,

220 comparisons being $24.1 \%, 16.2 \%, 39 \%$, and $31.6 \%$, respectively.

222 There is a notable difference in the number of DEGs called between VG and GV hybrids between 223 the R16A Clark and Wilson datasets. The R16A Clark data used an introgression strain of $N$. 224 giraulti, R16A, that has a nuclear genome derived from $N$. giraulti but maintains $N$. vitripennis 225 mitochondria, therefore the R16A Clark hybrids all have the same genetic makeup whereas the 226 Wilson reciprocal hybrids have the same nuclear genome but different cytoplasms; yet, we do 227 see eight genes called as differentially expressed between the VG and GV hybrids in the R16A 228 Clark data. Three of the eight genes in the R16A Clark data (LOC116416025, LOC116416106, 229 LOC116417553) were only called as differentially expressed between the VG and GV hybrids in 230 the R16A Clark dataset and weren't called as differentially expressed in the Wilson dataset. The 231 other five genes (LOC107981401, LOC100114950, LOC116415892, LOC103317241, 232 LOC107981942) were called as differentially expressed between the VG and GV in both datasets. 
233 In the Wilson data, we called 116 DEGs, 111 of which are uniquely to the Wilson data set. The

234 original Wang et al. 2016 publication did not investigate differential expression between the

235 hybrids [19]. Here we report a new way of looking at the data, and despite the same genetic

236 makeup between the hybrids in the R16A Clark data, we do observe differential expression

237 between the hybrids, and five of those eight genes are also called as differentially expressed in

238 the Wilson data.

239 Four (LOC107981401, LOC100114950, LOC116415892, and LOC103317241) out of the

240 five DEGs shared between the data sets are uncharacterized proteins located on Chr 1, Chr 2,

241 and Chr 4. To gain insight into the possible functions of these genes, we used NCBIs BLASTp

242 excluding Nasonia [29,30] to find regions of similarity between these sequences and

243 characterized sequences. We observe several significant hits to different insects including

244 Drosophila suggesting that these proteins have at least some conservation in insects over $>300$

245 million years. The fifth shared DEG, LOC107981942, located on chromosome 1, is annotated as

246 a zinc finger BED domain-containing protein 1. An NCBI Conserved Domain Search

247 (https://www.ncbi.nlm.nih.gov/Structure/cdd/wrpsb.cgi) using these protein sequences uncovered

248 no significant hits with LOC100114950, LOC116415892, and LOC103317241. However,

249 LOC107981401 and LOC107981942 show significant hits for transposase domain superfamilies

250 cl24015 and cl04853, respectively. The role of these proteins in Nasonia remains unclear.

\section{Lack of Parent-of-Origin effects in Nasonia hybrids}

252 We used allele-specific expression (ASE) analyses to detect parent-of-origin effects — indicated

253 by allelic bias - in Nasonia hybrids. The inference of genomic imprinting for each dataset was

254 limited to those sites that meet our filtering criteria (see Methods). We find 107,206 and 115,490

255 sites to be fixed and different between VV and GG samples, in the R16A Clark and Wilson

256 datasets, respectively. Limiting the analysis to only fixed and different sites, there are 6,377 and

2577,164 genes with at least 2 informative SNPs in the reciprocal hybrids in the R16A Clark data set 
258 and Wilson datasets, respectively. Using this approach, we find no evidence of genomic imprinting

259 in whole adult female samples of Nasonia in the R16A Clark data (Fig 4A). But for the Wilson

260 data we found two genes that show a pattern of expression consistent with genomic imprinting:

261 CPR35 and LOC103315494. In the VG hybrid, CPR35 shows a bias towards the paternally

262 inherited N. giraulti allele at an allele ratio of $65.3 \%$ and in the GV hybrid towards the paternally

263 inherited N. vitripennis allele, with an allele ratio of 62\% (S2 Table). CPR35 is a cuticular protein

264 in the RR family member 35. Similarly, LOC103315494 shows bias towards the paternally

265 inherited allele with allele ratios of $65.26 \%$ and $61.58 \%$ in VG and GV, respectively (S2 Table).

266 Although both imprinted genes, CPR35 and LOC103315494, fall below the mean depth and

267 average number of SNPs per gene, both genes are above the thresholds applied here (S3 Table). 


\section{A clark}

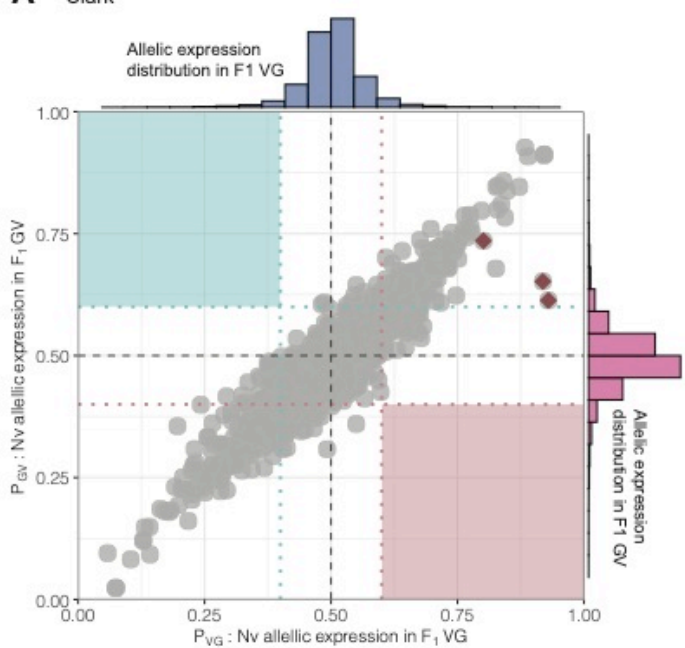

B Wilson
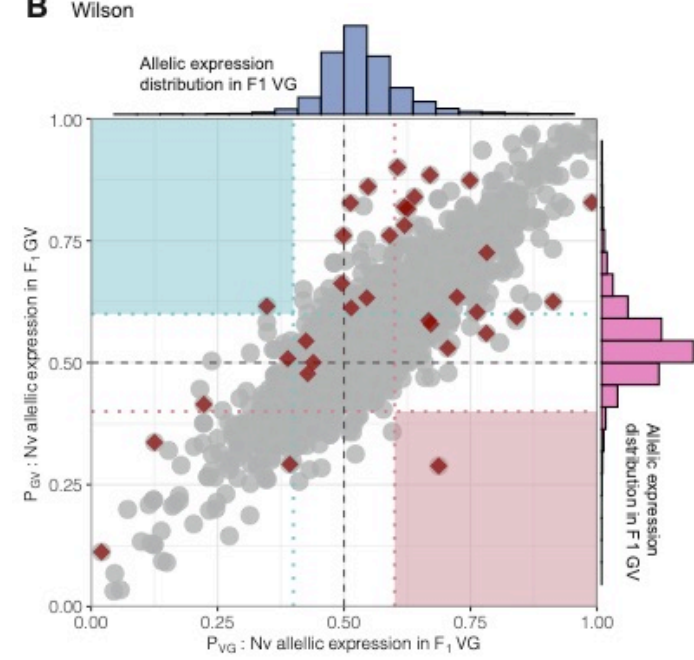

Fig 4. Scatterplots of the expression of the $N$. vitripennis alleles in the two reciprocal hybrids, VG

270 (x-axis) and GV (y-axis), in the R16A Clark (A) and Wilson (B) datasets. Genes with at least two

271 informative SNPs with a minimum depth of 30 were used (R16A Clark $=6,377$, Wilson $=7,164)$.

272 Genes exhibiting a significant difference in allelic bias between the hybrids (Fisher's exact test,

273 FDR-adj. $p<0.05$ ) are highlighted in red. Paternally imprinted genes are expected to appear in the

274 upper left corner (light blue box), and maternally imprinted genes in the lower right corner (light

275 pink box). Histograms of the $N$. vitripennis allele expression are shown for VG (blue) and GV 276 (pink). 


\section{Allele-specific expression differences in Nasonia hybrids}

278 We find three genes with higher expression of the $N$. vitripennis allele in both hybrids, in both 279 datasets, indicative of cis-regulatory effects. The genes LOC100123729, LOC100123734, and

280 LOC100113683 show consistent differences in allelic expression between VG and GV hybrids 281 (FDR- $p \leq 0.05$ ) in both datasets, but the ratio of the $N$. vitripennis allele differs between the hybrids 282 (S2 Table). In the R16A Clark dataset: LOC100123729 in the VG hybrids the N. vitripennis allele accounts for $93 \%$ of the reads, whereas in the GV hybrids this ratio is $61 \%$. In the Wilson dataset, both hybrids showed higher expression of the $N$. vitripennis allele. In the Wilson data, the $N$. vitripennis allele ratio was $61 \%$ in VG and $90 \%$ in GV. LOC100123729 is located on chromosome datasets show a higher expression for the N. vitripennis allele for LOC100123734. In the R16A Clark data, the ratio of the N. vitripennis allele in VG was $92 \%$ and in GV $65 \%$. In the Wilson data, the VG hybrids showed less expression for the $N$. vitripennis allele than the GV hybrids, at a ratio

292 of $64 \%$ and $84 \%$ of the reads, respectively. Finally, LOC100113683, which is located on

293 chromosome 4, and is annotated as a general odorant-binding protein 56d also shows more

294 expression for the $N$. vitripennis allele in both datasets and both hybrids $(80.13 \%$ and $73.54 \%$ for

295 VG and GV in R16A Clark, 78.22\% and 72.57\% in Wilson). Odorant binding proteins are thought

296 to be involved in the stimulation of the odorant receptors by binding and transporting odorants 297 which activate the olfactory signal transduction pathway [32].

\section{R16A strain retains $\mathbf{N}$. vitripennis alleles}

$299 \mathrm{R} 16 \mathrm{~A}$ is a strain produced by backcrossing an $N$. vitripennis female to an $N$. giraulti male and 300 repeating that for 16 generations [18]. This should give a complete $N$. giraulti nuclear genome 
with $N$. vitripennis mitochondria. However, we identified two regions in the R16A strain that still show $N$. vitripennis alleles and named them R16A non-introgressed locus 1 and R16A nonintrogressed locus 2 (S4 Table). Each region is identified by a single marker that retains the $N$.

304 vitripennis allele. Locus 1 contains 44 genes and Locus 2 contains 14 genes. Both of these 305 regions are found on Chromosome 1, and Locus 2 lies within the confidence intervals of the 306 mortality locus for N. vitripennis maternity hybrids identified by Niehuis et al. 2008 [21] (i.e., F2

307 recombinant hybrids with a $N$. vitripennis cytoplasm showed a significant transmission ratio 308 distortion at this region favoring the $N$. vitripennis allele). R16 A non-introgressed locus 1 harbors 309 a mitochondrial ribosomal gene (39 S ribosomal protein 38) which is a good candidate gene for 310 causing its retention in R16A despite intensive introgression. It would also explain the observed 311 nuc-cytoplasmic effect in F2 recombinant males in a vitripennis cytoplasm, despite the fact that 312 R16A was used as a giraulti parental line in Gadau et al. (1999) [20]. Gadau et al. interestingly 313 also mapped one of the nuc-cytoplasmic incompatibility loci to chromosome 1 (called LG1 in the 314 manuscript) [20]. Mutations in mitochondrial ribosomal proteins in humans have severe effects 315 [33].

318 We compared the location of genes with either significant differential gene expression or 319 significant differences in allele-specific expression between VG and GV hybrids to the location of 320 previously identified mortality-associated loci. Three of the five genes that were called as 321 differentially expressed between VG and GV hybrids in both the R16A Clark and Wilson data sets 322 (S5 Table) are located within mortality associated loci. LOC103317241 is located within a locus 323 on Chr 2 that is associated with mortality in VG hybrids, and LOC107981401 and LOC100114950 324 are within a locus on Chr 4 that is associated with mortality in GV hybrids. Moreover, two of the 
325 three genes showing consistent allele-specific expression in the two data sets are located near

326 one another in the mortality-associated locus on Chr. 2 (LOC100123729 and LOC100123734).

327 None of the genes that are differentially expressed or that exhibit allele-specific expression are

328 located within the 2 loci that retain the $N$. vitripennis genotype in the R16A Clark strain, nor did

329 we find any overlap of these gene sets with either the oxidative phosphorylation or the

330 mitochondrial ribosomal proteins.

\section{Discussion}

332 We successfully replicate the findings from Wang et al. 2016, showing a lack of parent-of-origin 333 effects in Nasonia transcriptomes [19]. This replication occurs independently in a different

334 laboratory, with different Nasonia individuals derived from a slightly different cross, different 335 bioinformatic pipelines, and sequencing technology. Our results from both the reanalyzed R16A 336 Clark and Wilson datasets could only demonstrate a species-of-origin effect but no parent-of337 origin effect within Nasonia F1 female hybrids, which may have explained the lack of mortality in 338 the F1 females relative to the F2 recombinant hybrid males. The larger number of differentially 339 expressed genes between the two parental species in our study relative to the Wang et al (2016) 340 [19] (1001 vs 799) is most likely the result of using a standard $N$. giraulti strain (RV2Xu) rather 341 than an introgression strain (R16A) where the nuclear genome of $N$. giraulti was introgressed into

342 a N. vitripennis cytoplasm. Additionally, we found genomic regions that resisted introgression in 343 the R16A Nasonia strains utilized by Wang et al. 2016 [19]. Furthermore, we present a 344 reproducible workflow for processing raw RNA sequence samples to call differential expression 345 and allele-specific expression openly available on the GitHub page: 346 https://github.com/SexChrLab/Nasonia. 
The primary difference between the R16A Clark cross and the Wilson cross is the N. giraulti strain choice Fig 1B. The new crosses presented here used the strain Rv2X(u), which is a pure $N$. giraulti strain that was used for sequencing the genome [17]. Wang et al. 2016 used an

352 introgression strain, R16A, which has a largely $N$. giraulti nuclear genome with an $N$. vitripennis 353 cytoplasm [19]. This strain was produced by mating an $N$. vitripennis female with an $N$. giraulti 354 male, and then repeatedly backcrossing the strain to $N$. giraulti males for a further 15 generations 355 [18]. Hence, both sets of hybrids should be heterozygous at every nuclear locus for species 356 specific markers (though see above for two non-introgressed regions); however, both reciprocal 357 R16A Clark hybrids have $N$. vitripennis mitochondria while the new hybrids have their maternal 358 species' mitochondria. This means that in addition to looking at parent-of-origin effects, our new crosses are uniquely suited to investigate allelic expression biases in the context of nuclearmitochondrial incompatibility and hybrid dysfunction.

\section{Observed differences in hybrids between data sets}

We observe substantially more DEGs between the hybrids, VG and GV, in the Wilson data set compared to the R16A Clark data set. The smaller number of DEGs detected in the R16A Clark data in this particular comparison is likely partially due to the one excluded $F_{1}$ GV sample (see Materials and methods). Another likely contributing factor is the differences in one parental strain between the Wilson and R16A Clark data sets. The Wilson data presented here consist of inbred parental $N$. vitripennis (strain AsymCX) VV and N. giraulti (strain RV2Xu) GG lines, and reciprocal

368 F1 crosses. This cross differs from the R16A Clark data, which used the same N. vitripennis strain

369 but rather than a normal $N$. giraulti strain they used the introgression strain, R16A, that has a 370 nuclear genome derived from $N$. giraulti and a cytoplasm/mitochondria derived from N. vitripennis 371 (see R16A section). Despite these differences, of the eight genes that are differentially expressed 372 between the VG and GV hybrids. five are shared between both data sets. Although we were not 373 specifically looking for this, we found that three of the five genes showing differential expression 
374 in both data sets as well as two of the three genes showing allele (species)-specific expression in

375 both data sets are located in previously identified loci that are associated with the observed F2

376 recombinant male hybrid breakdown from the same crosses [20,21]. These findings point towards

377 an involvement of cis regulatory elements in the genetic architecture of the F2-hybrid male

378 breakdown in Nasonia. The finding that, despite using different strains of wasps, we are still able

379 to identify genes associated with these hybrid defects, which bolsters our confidence in further

380 pursuing these genes in our investigation of the genetic architecture of hybrid barriers in Nasonia.

\section{The choice of reference and tools does not alter main findings}

382 The authors of the Wang et al. 2016 paper used different computational tools for trimming and

383 alignment than the current study [19]. Additionally, in Wang et al. 2016, the RNAseq reads were

384 aligned to both an N. vitripennis and N. giraulti reference genome [19]; whereas here, we created

385 a pseudo $N$. giraulti reference genome from the fixed and differentiated sites between the inbred

$386 N$. vitripennis and $N$. giraulti parental lines. Often, different tools and statistical approaches result

387 in different findings [34,35]; however, despite different approaches, we observe the same pattern

388 as what was originally reported in Wang et al. 2016 [19], a lack of parent-of-origin expression in

389 Nasonia.

\section{A reproducible workflow for investigating genomic imprinting}

391 Significant factors contributing to irreproducible research include selective reporting, unavailable

392 code and methods, low statistical power, poor experimental design, and raw data not available

393 from the original lab [23]. We replicate a robust experimental design (current study) initially

394 presented in the Wang et al. (2016) [19] and present a new workflow for calling DE and ASE in

395 those two independent but analog Nasonia datasets. Both datasets are publicly available for

396 download on the short read archive (SRA) PRJNA260391 and PRJNA613065, respectively. In

397 our analyses of the Wilson data and reanalysis of the R16A data, we corroborated the original 
findings from Wang et al. 2016 [19]. There are no parent-of-origin effects in Nasonia. All dependencies for data processing are provided as a Conda environment, allowing for seamless replication. All code is openly available on GitHub https://github.com/SexChrLab/Nasonia.

\section{Materials and methods}

\section{Nasonia vitripennis and Nasonia giraulti inbred and reciprocal F1 hybrid datasets}

403 RNA sequence (RNAseq) samples for 4 female samples each from parental species, $N$. 404 vitripennis (VV) and $N$. giraulti (GG), and from each reciprocal $F 1$ cross $\left(F_{1} V G\right.$, female hybrids with $N$. vitripennis mothers, and $\mathrm{F}_{1} \mathrm{GV}$, female hybrids with $N$. giraulti mothers), as shown in Fig 1A, were obtained from a 2016 publication [19] from SRA PRJNA299670. We refer to the data from [19] as R16A Clark. One $F_{1}$ GV RNAseq sample from the R16A Clark dataset (SRR2773798) was excluded due to low quality, as in the original publication [19].

The newly generated crosses consisted of 12 RNAseq samples of inbred isofemale lines of

411 parental N. vitripennis (strain AsymCX) VV and N. giraulti (strain RV2Xu) GG lines, and reciprocal

$412 \mathrm{~F} 1$ crosses $F_{1} V G$, and $F_{1} G V$. (Fig 1A). Whole transcriptome for these samples is available on 413 SRA PRJNA613065. This cross differs from the R16A Clark data, which used the same $N$. 414 vitripennis strain but rather than a standard $N$. giraulti strain used an introgression strain, R16A, 415 that has a nuclear genome derived from N. giraulti and a cytoplasm/mitochondria derived from $N$. 416 vitripennis (see R16A section below) Fig 1B. Total RNA was extracted from a pool of four 48 hour 417 post-eclosion adult females using a Qiagen RNeasy Plus Mini kit (Qiagen, CA). RNA-seq libraries 418 were prepared with $2 \mu \mathrm{g}$ of total RNA using the Illumina Stranded mRNA library prep kit and were 419 sequenced on a HiSeq2500 instrument following standard Illumina protocols. Three biological 420 replicates were generated for each parent and hybrid, with 100-bp paired-end reads per replicate. 
421 Sample IDs, parent cross information, and SRA bioproject accession numbers for R16A Clark

422 and Wilson datasets are listed in S1 Table.

\section{Quality control}

424 Raw sequence data from both datasets were processed and analyzed according to the workflow

425 presented in Fig 1C. The quality of the FASTQ files was assessed before and after trimming using

426 FastQC v0.11 [36] and MultiQC v1.0 [37]. Reads were trimmed to remove bases with a quality

427 score less than 10 for the leading and trailing stand, applying a sliding window of 4 with a minimum

428 mean PHRED quality of 15 in the window and a minimum read length of 80 bases, and adapters

429 were removed using Trimmomatic v0.36 [38]. Pre- and post-trimming multiQC reports for the

430 R16A Clark and Wilson datasets are available on the GitHub page:

431 https://github.com/SexChrLab/Nasonia.

\section{Variant calling}

433 For variant calling, BAM files were preprocessed by adding read groups with Picard's

434 AddOrReplaceReadGroups and by marking duplicates with Picard's MarkDuplicates

435 (https://github.com/broadinstitute/picard). Variants were called using GATK [39-41] and the

436 scatter-gather approach: Sample genotype likelihoods were called with HaplotypeCaller minimum

437 base quality of 2 . The resulting gVCFs were merged with CombineGVCFs, and joint genotyping

438 across all samples was carried out with GenotypeGVCFs with a minimum confidence threshold 439 of 10.

\section{Pseudo $N$. giraulti reference genome assembly}

441 To create a pseudo $N$. giraulti reference genome, fixed differences in the homozygous $N$. giraulti

442 and $N$. vitripennis variant call file (VCF) files were identified using a custom Python script, 
443 available on the GitHub page: https://github.com/SexChrLab/Nasonia. Briefly, a site was

444 considered to be fixed and different if it was homozygous for the $N$. vitripennis reference allele

445 among all three of the biological VV samples and homozygous alternate among all three of the

446 biological GG samples. Only homozygous sites were included, as the $N$. giraulti and N. vitripennis

447 lines are highly inbred. The filtered sites were then used to create a pseudo N. giraulti reference

448 sequence with the FastaAlternateReferenceMaker function in GATK version 3.8 (available at:

449 http://www.broadinstitute.org/gatk/). Reference bases in the N. vitripennis genome were replaced

450 with the alternate SNP base at variant positions. Following a similar protocol for comparison, we

451 now aligned reads in each sample to the pseudo $N$. giraulti genome reference with HISAT2

452 version 2.1.0, and performed identical preprocessing steps prior to variant calling with GATK

453 version 3.8 HaplotypeCaller.

\section{RNAseq alignment and gene expression level quantification}

455 Trimmed sequence reads were mapped to the NCBI N. vitripennis reference genome (assembly 456 accession GCF_009193385.2), as well as the pseudo N. giraulti reference using HISAT2 [42].

457 The resulting SAM sequence alignment files were converted to BAM, and coordinates were sorted 458 and indexed with samtools 1.8 [43]. RNAseq read counts were quantified from the N. vitripennis 459 as well as the custom $N$. giraulti alignments using Subread featureCounts [44] with the $N$. 460 vitripennis gene annotation.

\section{Inference of differential gene expression}

462 Differential expression (DE) analyses were carried out by linear modeling as implemented in the

$463 \mathrm{R}$ package limma [45]. An average of the reads mapped to each gene in the $N$. vitripennis and

464 the pseudo N. giraulti genome references were used in the DE analyses. Counts were filtered to 465 remove lowly expressed genes by retaining genes with a mean FPKM $\leq 0.5$ in at least one sample 466 group (VV, GG, VG, or GV). Normalization of expression estimates was accomplished by 
calculating the trimmed mean of M-values (TMM) with edgeR [46]. The voom method [47] was

then employed to normalize expression intensities by generating a weight for each observation.

Gene expression is then reported as log counts per million (logCPM). Gene expression correlation

470 between datasets and between species within each dataset was assessed using Pearson's

471 correlation of mean logCPM values of each gene. Dimensionality reduction of the filtered and

472 normalized gene expression data was carried out using scaled and centered PCA with the

$473 \operatorname{prcomp}()$ function of base R. Differential expression analysis with voom was carried out for each

474 pairwise comparison between strains (VV, GG, VG, and GV) for each data set. We identified

475 genes that exhibited significant expression differences with an adjusted $p$-value of $\leq 0.01$ and an

476 absolute $\log _{2}$ fold-change $\left(\log _{2} \mathrm{FC}\right) \leq 2$.

\section{Analysis of allele-specific expression in reciprocal F1 hybrids}

478 Allele-specific expression (ASE) levels were obtained using GATK ASEReadCounter [40] with a

479 minimum mapping quality of 10 , minimum base quality of 2 , and a minimum depth of 30 . Only

480 sites with a fixed difference between inbred VV and GG for both R16A Clark and Wilson datasets

481 were used for downstream analysis of allele-specific expression. Allele counts obtained from

482 GATK ASEReadCounter were intersected with the $N$. vitripennis gene annotation file using

483 bedtools version 2.24.0 [48]; the resulting output contained allele counts for each SNP and

484 corresponding gene information. The F1 hybrids' allele counts with gene information was read

485 into R and then filtered to only include genes with at least two SNPs with minimum depth of 30.

486 We counted the number of allele-counts for the reference allele $(N$. vitripennis $)$ and alternative $(N$.

487 giraulti) allele at polymorphic SNP positions. We quantified the number of SNPs in each hybrid

488 replicate that 1 ) showed a bias towards the allele that came from the $N$. vitripennis parent, 2)

489 showed a bias towards the allele that came from the $N$. giraulti parent, and 3) showed no

490 difference (ND) in an expression of its parental alleles. The significance of allelic bias was

491 determined using Fisher's exact test. Significant genes were selected using a Benjamini- 
Hochberg false discovery rate FDR-adjusted $p$-value threshold of 0.05 . As Nasonia are haplodiploid, all ASE analyses were carried out on the diploid female hybrids.

\section{Identifying loci associated with hybrid mortality}

495 Nasonia recombinant F2 hybrid males (haploid sons of F1 female hybrids) suffer mortality during

496 development that differs between VG and GV hybrids [18]. Niehuis et al. 2008 identified four

497 genomic regions associated with this mortality (i.e., regions in which one parent species' alleles

498 are underrepresented due to mortality during development); three are associated with mortality in

499 hybrids with $N$. vitripennis maternity and one is associated with hybrids with $N$. giraulti maternity

500 [21]. Gibson et al. 2013 later identified a second locus related to mortality in the hybrids with $N$.

501 giraulti maternity [22]. Given that the F2 hybrid females analyzed here experience far less

502 mortality than their haploid male offspring, we hypothesized that these diploid females may use

503 biased allelic expression to rescue themselves from the mortality. To compare our results with

504 these previous studies, we had to map the previous loci to the latest Nasonia assembly (PSR1.1,

505 [49]). Niehuis et al. 2008 defined their candidate loci based on the genetic distance along the

506 chromosome (centimorgans) [21]. The physical locations of the markers along the chromosomes

507 were later identified by Niehius et al. 2010 [50]. Using the genetic distances between these

508 markers in both the 2008 and 2010 Niehuis et al. studies [21,50], we calculated the conversion

509 ratio between the genetic distances in these two studies (S6 Table). We then converted those

5102008 genetic distances that correspond to the $95 \%$ Confidence Intervals for these loci to the

511 genetic distances reported by Niehuis et al. 2010 [50], which used an Illumina Goldengate

512 Genotyping Array (Illumina Inc., San Diego, USA) to produce a more complete and much higher

513 resolution genetic map of Nasonia. This array uses Single Nucleotide Polymorphisms (SNPs) to

514 genotype samples at $\sim 1500$ loci, which allowed us to identify SNP markers that closely bound the

515 mortality loci from the 2008 study. Gibson et al. 2013 used the same genotyping array, so this

516 conversion was unnecessary for converting the second mortality locus in $N$. giraulti maternity 
517 hybrids [22]. We used the 100bp of sequence flanking each SNP marker to perform a BLAST

518 search of the PSR1.1 assembly and to identify their positions. We then used all of the PSR1.1

519 annotated genes within these loci to look for enrichment of genes showing biased expression.

520 Mortality loci and genomic location are reported in S4 Table.

\section{Additional gene categories of interest}

522 Previous work has identified potential classes of genes that may be involved in nuclear-

523 mitochondrial incompatibilities in Nasonia, the oxidative phosphorylation genes [51] and the

524 mitochondrial ribosomal proteins [52]. We used the annotated gene sets from these studies to 525 test for enrichment of genes with biased allelic expression. Lists of the genes of interest and their 526 genomic location is reported in S4 Table.

\section{Analysis of R16A strain}

528 In order to assess whether the introgression of the $N$. giraulti nuclear genome into the R16A Clark

529 strain is complete, we analyzed two samples of the R16A strain using the Illumina Goldengate

530 Genotyping Array used in Niehuis et al. 2010 [50]. We searched for SNP markers that retained

531 the $N$. vitripennis allele and only considered markers that consistently identified the proper allele

532 in both parent species controls and that were consistent across both R16A samples, leaving 1378

533 markers. We defined a locus as all of the sequences between the two markers that flank a marker

534 showing the $N$. vitripennis allele (S2 Table). As above, we performed a BLAST search of the

535 PSR1.1 assembly to identify the positions of these markers. We identified all genes from the

536 PSR1.1 assembly that lie between the flanking markers and further analyzed their expression

537 patterns.

539 Scripts and gene lists used to analyze these data are publicly available on GitHub, 


\section{Acknowledgments}

542 The authors acknowledge Research Computing at Arizona State University for providing HPC

543 resources that have contributed to the research results reported within this paper.

\section{Funding Disclosure}

545 ARCS Spetzler Scholar additionally supported KCO. HMN was supported by an ASU Center for

546 Evolution and Medicine postdoctoral fellowship. MAW was supported by the National Institute of

547 General Medical Sciences (NIGMS) of the National Institutes of Health (NIH) grant

548 R35GM124827. JDG was supported by the Division of Integrative Organismal Systems (IOS) of

549 the National Science Foundation (NSF) grant 1145509 and by research funds provided by

550 Georgia Southern University. JG was in part supported by a grant from the German Research

551 Foundation (DFG) to N.S. (281125614/GRK2220, Project B7). The funders had no role in study

552 design, data collection and analysis, decision to publish, or preparation of the manuscript. 


\section{Supplemental Data}

S1 Fig. Volcano plots for differential expression and venn diagram of DEGs between the datasets when taking the average of the counts when aligned to $N$. vitripennis and to pseudo $\mathbf{N}$. giraulti reference genome. Volcano plots of DEGs detected between the different comparisons involving $N$. vitripennis, $N$. giraulti, and the two reciprocal $F_{1}$ hybrids in the R16A Clark (left side) and Wilson (right side) datasets. Venn diagrams of the overlap of significant DEGs in each comparison is shown.

S1 Table. Sample identifiers. The samples for each dataset used in the project are provided here. Samples from this study are uploaded at https://www.ncbi.nlm.nih.gov/sra/PRJNA613065.

S2 Table. Allele-specific expression differences between hybrids. The number of allelecounts for the reference allele ( $N$. vitripennis) and alternative $(N$. giraulti) allele at polymorphic SNPs within a gene. Minimum of two SNPs for a gene to be included. The significance of allelic bias was determined using Fisher's exact test. Significant genes were selected using a BenjaminiHochberg false discovery rate FDR-adjusted $p$-value threshold of 0.05 .

S3 Table. Mean and median allele and gene depth for Wilson dataset. Mean and median

572 allele and gene depth for each GV and VG sample in the Wilson data set. Number of SNPs for all 573 genes, CPR35, and LOC103315494.

575 S4 Table. Genomic location of mortality loci and gene sets of interest. Previously reported

576 loci associated with mortality in Nasonia hybrids. 95\% Confidence Intervals of loci identified in

577 Niehuis et al. 2008 were converted to genetic distances along the chromosomes and the closest

578 SNP markers from Niehius et al. 2010 were identified $[21,50]$. SNP markers for the locus identified 
579 in Gibson et al. 2013 were used directly [22]. The SNP marker locations in the PSR1.1 assembly

580 were found via BLAST and all genes within the bounds of these markers are included. The two

581 non-introgressed regions from the R16A strain are included as well as genes from two

582 mitochondria-associated pathways, the oxidative phosphorylation pathway [51] and the

583 mitochondrial ribosomal proteins [52].

584

585 S5 Table. Directional bias of differentially expressed genes between VG and GV in Clark

586 and Wilson datasets. Five genes that were called as differentially expressed between VG and

587 GV hybrids in both the Clark and Wilson data sets.

588

58956 Table. Locus conversion calculations. Calculations for converting the genetic map

590 positions (centimorgan, cM) of mortality loci identified by Niehuis et al. 2008 to the physical

591 chromosomal positions of the latest genome assembly (PSR1.1) [21]. 


\section{References}

594

595

596

597

598

599

600

601

602

603

604

605

606

607

608

609

610

611

612

613

614

615

616

617

618

619

620

621

622

623

624

625

626

627

628

1. Reik W, Walter J. Genomic imprinting: parental influence on the genome. Nature Reviews Genetics. 2001. pp. 21-32. doi:10.1038/35047554

2. Ishida M, Moore GE. The role of imprinted genes in humans. Mol Aspects Med. 2013;34: 826-840.

3. Isles AR, Davies W, Wilkinson LS. Genomic imprinting and the social brain. Philos Trans R Soc Lond B Biol Sci. 2006;361: 2229-2237.

4. Moore T, Haig D. Genomic imprinting in mammalian development: a parental tug-of-war. Trends Genet. 1991;7: 45-49.

5. Lawson HA, Cheverud JM, Wolf JB. Genomic imprinting and parent-of-origin effects on complex traits. Nat Rev Genet. 2013;14: 609-617.

6. Yan H, Bonasio R, Simola DF, Liebig J, Berger SL, Reinberg D. DNA methylation in social insects: how epigenetics can control behavior and longevity. Annu Rev Entomol. 2015;60: 435-452.

7. Queller DC. Theory of genomic imprinting conflict in social insects. BMC Evol Biol. 2003;3: 15.

8. Haig D. Intragenomic conflict and the evolution of eusociality. J Theor Biol. 1992;156: 401403.

9. Galbraith DA, Kocher SD, Glenn T, Albert I, Hunt GJ, Strassmann JE, et al. Testing the kinship theory of intragenomic conflict in honey bees (Apis mellifera). Proc Natl Acad Sci U S A. 2016;113: 1020-1025.

10. Smith NMA, Yagound B, Remnant EJ, Foster CSP, Buchmann G, Allsopp MH, et al. Paternally-biased gene expression follows kin-selected predictions in female honey bee embryos. Mol Ecol. 2020;29: 1523-1533.

11. Kocher SD, Tsuruda JM, Gibson JD, Emore CM, Arechavaleta-Velasco ME, Queller DC, et al. A Search for Parent-of-Origin Effects on Honey Bee Gene Expression. G3 . 2015;5: 1657-1662.

12. Gibson JD, Arechavaleta-Velasco ME, Tsuruda JM, Hunt GJ. Biased Allele Expression and Aggression in Hybrid Honeybees may be Influenced by Inappropriate Nuclear-Cytoplasmic Signaling. Frontiers in Genetics. 2015. doi:10.3389/fgene.2015.00343

13. Hunt GJ, Guzmán-Novoa E, Fondrk MK, Page RE Jr. Quantitative trait loci for honey bee stinging behavior and body size. Genetics. 1998;148: 1203-1213.

14. Hunt GJ. Flight and fight: A comparative view of the neurophysiology and genetics of honey bee defensive behavior. J Insect Physiol. 2007;53: 399-410.

15. Shorter JR, Arechavaleta-Velasco M, Robles-Rios C, Hunt GJ. A Genetic Analysis of the Stinging and Guarding Behaviors of the Honey Bee. Behavior Genetics. 2012. pp. 663- 
16. Alaux C, Sinha S, Hasadsri L, Hunt GJ, Guzmán-Novoa E, DeGrandi-Hoffman G, et al. Honey bee aggression supports a link between gene regulation and behavioral evolution. Proc Natl Acad Sci U S A. 2009;106: 15400-15405.

17. Werren JH, Richards S, Desjardins CA, Niehuis O, Gadau J, Colbourne JK, et al. Functional and evolutionary insights from the genomes of three parasitoid Nasonia species. Science. 2010;327: 343-348.

18. Breeuwer JAJ, Werren JH. HYBRID BREAKDOWN BETWEEN TWO HAPLODIPLOID SPECIES: THE ROLE OF NUCLEAR AND CYTOPLASMIC GENES. Evolution. 1995;49: 705-717.

19. Wang X, Werren JH, Clark AG. Allele-Specific Transcriptome and Methylome Analysis Reveals Stable Inheritance and Cis-Regulation of DNA Methylation in Nasonia. PLOS Biology. 2016. p. e1002500. doi:10.1371/journal.pbio.1002500

20. Gadau J, Page RE Jr, Werren JH. Mapping of hybrid incompatibility loci in Nasonia. Genetics. 1999;153: 1731-1741.

21. Niehuis O, Judson AK, Gadau J. Cytonuclear genic incompatibilities cause increased mortality in male F2 hybrids of Nasonia giraulti and N. vitripennis. Genetics. 2008;178: 413426.

22. Gibson JD, Niehuis O, Peirson BRE, Cash El, Gadau J. Genetic and developmental basis of F2 hybrid breakdown in Nasonia parasitoid wasps. Evolution. 2013;67: 2124-2132.

23. Baker M. 1,500 scientists lift the lid on reproducibility. Nature. 2016. pp. 452-454. doi:10.1038/533452a

24. Casadevall A, Fang FC. Reproducible science. Infect Immun. 2010;78: 4972-4975.

25. Baker M. Irreproducible biology research costs put at $\$ 28$ billion per year. Nature. 2015;533. Available: http://www.target-biomed.de/resources//rreproducible-biologyresearch.pdf

26. Freedman LP, Inglese J. The Increasing Urgency for Standards in Basic Biologic Research. Cancer Research. 2014. pp. 4024-4029. doi:10.1158/0008-5472.can-14-0925

27. Simoneau J, Dumontier S, Gosselin R, Scott MS. Current RNA-seq methodology reporting limits reproducibility. Brief Bioinform. 2019. doi:10.1093/bib/bbz124

28. Su Z, Łabaj PP, Li S, Thierry-Mieg J, Thierry-Mieg D, Shi W, et al. A comprehensive assessment of RNA-seq accuracy, reproducibility and information content by the Sequencing Quality Control Consortium. Nat Biotechnol. 2014;32: 903-914.

29. Johnson M, Zaretskaya I, Raytselis Y, Merezhuk Y, McGinnis S, Madden TL. NCBI BLAST: a better web interface. Nucleic Acids Research. 2008. pp. W5-W9. doi:10.1093/nar/gkn201

30. NCBI Resource Coordinators, NCBI Resource Coordinators. Database Resources of the National Center for Biotechnology Information. Nucleic Acids Research. 2017. pp. D12D17. doi:10.1093/nar/gkw1071 
667

668

669

670

671

672

673

674

675

676

677

678

679

680

681

682

683

684

685

686

687

688

689

690

691

692

693

694

695

696

697

698

699

700

701

702

703

704

31. Tian C, Wang L, Ye G, Zhu S. Inhibition of melanization by a Nasonia defensin-like peptide: implications for host immune suppression. J Insect Physiol. 2010;56: 1857-1862.

32. He $Y$, Wang $K$, Zeng $Y$, Guo Z, Zhang $Y, W u ~ Q$, et al. Analysis of the antennal transcriptome and odorant-binding protein expression profiles of the parasitoid wasp Encarsia formosa. Genomics. 2020. pp. 2291-2301. doi:10.1016/j.ygeno.2019.12.025

33. Sylvester JE, Fischel-Ghodsian N, Mougey EB, O'Brien TW. Mitochondrial ribosomal proteins: candidate genes for mitochondrial disease. Genet Med. 2004;6: 73-80.

34. Schaarschmidt S, Fischer A, Zuther E, Hincha DK. Evaluation of Seven Different RNA-Seq Alignment Tools Based on Experimental Data from the Model Plant Arabidopsis thaliana. Int J Mol Sci. 2020;21. doi:10.3390/ijms21051720

35. Del Fabbro C, Scalabrin S, Morgante M, Giorgi FM. An extensive evaluation of read trimming effects on Illumina NGS data analysis. PLoS One. 2013;8: e85024.

36. Andrews S. FastQC: A Quality Control Tool for High Throughput Sequence Data [Online]. Online. 2010. Available: http://www.bioinformatics.babraham.ac.uk/projects/fastqc/

37. Ewels P, Magnusson M, Lundin S, Käller M. MultiQC: summarize analysis results for multiple tools and samples in a single report. Bioinformatics. 2016;32: 3047-3048.

38. Bolger AM, Lohse M, Usadel B. Trimmomatic: a flexible trimmer for Illumina sequence data. Bioinformatics. 2014;30: 2114-2120.

39. Van der Auwera GA, Carneiro MO, Hartl C, Poplin R, Del Angel G, Levy-Moonshine A, et al. From FastQ data to high confidence variant calls: the Genome Analysis Toolkit best practices pipeline. Curr Protoc Bioinformatics. 2013;43: 11.10.1-33.

40. McKenna A, Hanna M, Banks E, Sivachenko A, Cibulskis K, Kernytsky A, et al. The Genome Analysis Toolkit: a MapReduce framework for analyzing next-generation DNA sequencing data. Genome Res. 2010;20: 1297-1303.

41. DePristo MA, Banks E, Poplin R, Garimella KV, Maguire JR, Hartl C, et al. A framework for variation discovery and genotyping using next-generation DNA sequencing data. Nat Genet. 2011;43: 491-498.

42. Kim D, Langmead B, Salzberg SL. HISAT: a fast spliced aligner with low memory requirements. Nat Methods. 2015;12: 357-360.

43. Li H, Handsaker B, Wysoker A, Fennell T, Ruan J, Homer N, et al. The Sequence Alignment/Map format and SAMtools. Bioinformatics. 2009;25: 2078-2079.

44. Liao Y, Smyth GK, Shi W. featureCounts: an efficient general purpose program for assigning sequence reads to genomic features. Bioinformatics. 2014;30: 923-930.

45. Ritchie ME, Phipson B, Wu D, Hu Y, Law CW, Shi W, et al. limma powers differential expression analyses for RNA-sequencing and microarray studies. Nucleic Acids Res. 2015;43: e47.

46. Robinson MD, McCarthy DJ, Smyth GK. edgeR: a Bioconductor package for differential expression analysis of digital gene expression data. Bioinformatics. 2010;26: 139-140. 
47. Law CW, Chen Y, Shi W, Smyth GK. voom: precision weights unlock linear model analysis tools for RNA-seq read counts. Genome Biol. 2014;15: R29.

48. Quinlan AR, Hall IM. BEDTools: a flexible suite of utilities for comparing genomic features. Bioinformatics. 2010. pp. 841-842. doi:10.1093/bioinformatics/btq033

49. Benetta ED, Antoshechkin I, Yang T, Nguyen HQM, Ferree PM, Akbari OS. Genome Elimination Mediated by Gene Expression from a Selfish Chromosome. doi:10.1101/793273

50. Niehuis O, Gibson JD, Rosenberg MS, Pannebakker BA, Koevoets T, Judson AK, et al. Recombination and its impact on the genome of the haplodiploid parasitoid wasp Nasonia. PLoS One. 2010;5: e8597.

51. Gibson JD, Niehuis O, Verrelli BC, Gadau J. Contrasting patterns of selective constraints in nuclear-encoded genes of the oxidative phosphorylation pathway in holometabolous insects and their possible role in hybrid breakdown in Nasonia. Heredity . 2010;104: 310317.

52. Burton RS, Barreto FS. A disproportionate role for mtDNA in Dobzhansky-Muller incompatibilities? Molecular Ecology. 2012. pp. 4942-4957. doi:10.1111/mec.12006 